\title{
Aspectos básicos en la producción de semilla artesanal de maíz amilaceo
}

Basic aspects in the starchy corn handcrafted seed production

${ }^{1,2}$ Pedro José García Mendoza ${ }^{\mathrm{a}}{ }^{3}$ Rubén José Silva Diaz ${ }^{\mathrm{a}}$

\section{RESUMEN}

La agricultura se considera la base para el sustento de la población humana en el planeta tierra, por ser la responsable de la alimentación de toda su población, además de proveerle las fibras y demás materiales básicos para su sustento y proporcionarle el alimento para los animales de cría. La semilla constituye el factor más determinante en todo proceso agrícola, puesto que representa el máximo potencial genético desarrollado por la ciencia en la producción de los diferentes rubros agrícolas. En lo que concierne al maíz amiláceo, este cultivo representa a uno de los principales alimentos de los habitantes de la sierra peruana, siendo considerado un rubro estratégico para la seguridad alimentaria, debido a la gran relevancia social, económica, cultural y estratégica que tiene para su población. En este sentido, el presente trabajo tiene como objetivo general servir como guía técnica para orientar a los actores involucrados con la producción de semilla de maíz amiláceo de alta calidad en el Perú, pudiendo también servir para la producción de semilla de maíz amiláceo en toda la zona andina de Suramérica. El mismo ha sido elaborado considerando los aspectos técnicos y legales que rigen esta materia en el Perú, pero al mismo tiempo también observando las recomendaciones técnicas para la producción de semilla de maíz de alta calidad, establecidas por organismos internacionales de gran trayectoria en el cultivo, tales como el CIMMYT, la FAO, entre otros, además de recoger algunas de las experiencias personales en el cultivo de maíz y haciendo hincapié en que las etapas del procesamiento de las semillas son actividades que están bien estandarizadas en el ámbito internacional.

Palabras clave: semilla, maíz amiláceo, seguridad alimentaria

\section{ABSTRACT}

Agriculture is considered the basis for the livelihood of the human population on planet earth, because it is responsible for its population's feeding, in addition to providing the fibers and other basic materials for their livelihood and providing food for the animals of breeding. The seed is the most determining factor for any agricultural production activity, since it symbolizes the maximum genetic potential developed by science in the different crops agricultural. With regard to starchy corn, this crop represents one of the main foods of the Peruvian highlands inhabitants, being considered a strategic crop for food security, due to the great social, economic, cultural and strategic relevance for population. Thus, this work has main aimed to serve as a technical guide in order to help the actors involved with the 
production of the Peru high quality starch corn seed, and can also be used for starch corn seed producer throughout the Andean area of South America. The work has been prepared considering the technical and legal aspects that govern this matter in Peru, but at the same time also observing the technical recommendations for the high quality corn seed production, established by international organizations whit a long history in this crop, such as CIMMYT, FAO, among others, in addition to collecting some personal experiences in the corn cultivation and highlighting that the seed processing stages are activities that are well standardized in the international arena.

Keyword: seed, starchy corn, food security

${ }^{1}$ Universidad Nacional Autónoma de Tayacaja (UNAT) Perú.

${ }^{2}$ Investigador en Mejoramiento Genético, Rubro Maíz, Instituto Nacional de Investigaciones Agrícolas (INIA), Portuguesa, Venezuela.

${ }^{3}$ Investigador en Mejoramiento Genético, Rubro Maíz, Instituto Nacional de Investigaciones Agrícolas (INIA), CENIAP, Maracay, Venezuela.

${ }^{a}$ Ingeniero Agrónomo. 


\section{INTRODUCCIÓN}

La semilla se considera un factor esencial para la supervivencia de la humanidad, por cuanto almacena el potencial genético más alto desarrollado por la ciencia para la producción de los cultivos. De acuerdo a Oropeza et al. (2000), el cultivo de plantas es la base de la alimentación humana, al proveer a la humanidad los granos, frutos, vegetales y fibras, además de proporcionarle el alimento para los animales de cría, donde la semilla representa un elemento vital de la agricultura moderna, puesto que nueve de cada diez renglones alimenticios, disponibles en la actualidad, comienzan con la siembra de la semilla.

El agricultor moderno ha entendido muy bien que una buena cosecha comienza con una buena semilla. El avance de nuevos conocimientos científicos y tecnológicos obtenidos en diferentes áreas de la ciencia agrícola, se ha incorporado para desarrollar tecnologías de producción de semilla de alta calidad. En consecuencia, la semilla es el producto de numerosos aportes realizados por científicos agrícolas, donde destacan los genetistas, fitopatólogos, entomólogos, fisiólogos y agrónomos, quienes han logrado determinar los factores que mejoran la producción de las plantas (Oropeza, et al., 2000).

Los agricultores cada día están más conscientes de la importancia de utilizar semilla de alta calidad, sana, vigorosa, capaz de producir una plántula libre de enfermedades, lo que ha llevado a que la producción de semilla haya adquirido un grado de organización y especialización con visión empresarial, permitiendo que este insumo, estratégico para el sector agrícola, sea una actividad desarrollada bajo la cooperación del sector público y privado, donde se han involucrado los productores organizados, empresas semilleristas y demás actores de la cadena del sector agrícola.

El presente trabajo tiene como objetivo general servir como guía técnica para orientar a todos los actores involucrados con la producción de semilla de maíz amiláceo de alta calidad en el Perú. El mismo ha sido elaborado considerando los aspectos técnicos y legales que rigen esta materia en el Perú, pero al mismo tiempo también observando las recomendaciones técnicas para la producción de semilla de maíz de alta calidad, establecidas por organismos internacionales de gran trayectoria en el cultivo, tales como el CIMMYT, la FAO, entre otros, así como también la experiencia personal de los autores del trabajo.

Proceso de producción de semilla certificada de maíz en el Perú: En el Perú, el Ministerio de Agricultura a través del Instituto Nacional de Innovación Agraria (INIA), Autoridad en Semillas y en trabajo conjunto con los agricultores, productores y comerciantes de semillas, 
investigadores y organismos certificadores, ha desarrollado el Reglamento de la Ley General de Semillas, que ha sido aprobado mediante Decreto Supremo No 006-2012-AG (El Peruano, 2008). Este Reglamento está orientado a tener un marco jurídico moderno e integrador, cuyo principal objetivo es fortalecer la actividad de la Autoridad en Semillas y otorgar transparencia y seguridad para los usuarios, que coadyuve a la mejora de la disponibilidad de semilla en cantidad, calidad y oportunidad en apoyo de la competitividad las agroexportaciones.

Esta norma ha incorporado el interés de reconocer diferentes sistemas de producción como el tradicional o biodiverso. De esta manera, la legislación en semillas permite desarrollar una reglamentación específica para elevar la competitividad de los pequeños agricultores y se orienta a asegurar un uso sostenible de los recursos fitogenéticos y la conservación de la biodiversidad agrícola, con el objetivo de insertar a un sector importante del país al sistema formal de producción de semillas. Adicionalmente, el Reglamento de la Ley General de Semillas introduce las disposiciones necesarias para fomentar y potenciar una mayor participación del sector privado en la investigación, producción y comercialización de semillas, así como en la prestación de los servicios de análisis de calidad. Ello ampliará la base de usuarios de semillas, mejorando la competitividad de la agricultura nacional (MINAGRI, 2012). De acuerdo al MINAGRI (2012), el Programa Especial de la Autoridad en Semillas (PEAS) de la Dirección de Extensión Agraria, está encargada de la difusión de la norma y de absolver las dudas que puedan presentarse en su aplicación.

El Art. 5 del reglamento de la Ley General de Semillas, establece que la Autoridad en Semillas Corresponde al Instituto Nacional de Innovación Agraria (INIA), Organismo Público del Ministerio de Agricultura, quien debe ejercer las funciones de la Autoridad en Semillas, de acuerdo al artículo $6^{\circ}$ de la Ley (El Peruano, 2008; MINAGRI,2012). En este sentido, conforme a este reglamento, las funciones del INIA, como Autoridad en Semillas son:

- Normar y supervisar la producción, certificación y comercialización de semillas.

- Detectar y sancionar las infracciones, de conformidad con lo dispuesto en la Ley, el presente Reglamento y demás normas en materia de semillas.

- Llevar los Registros establecidos por la Ley.

- Verificar y homologar las categorías y calidad de las semillas importadas de acuerdo con lo establecido en la Ley y sus reglamentos.

- Promover, en coordinación con instituciones públicas y privadas, la utilización de semillas de buena calidad. 
Delegar o autorizar el servicio de certificación de semillas, a las entidades públicas o privadas que califiquen para tal actividad. En los lugares donde no existan dichas entidades, la certificación deberá efectuarla la Autoridad en Semillas.

- Otras que le sean encomendadas en concordancia con la Ley.

Categorías de semillas: A principios del pasado siglo, las estaciones experimentales de Estados Unidos y de Europa distribuyeron entre agricultores de la región sus primeras porciones de semilla obtenida de variedades mejoradas, con el propósito de que las multiplicaran y continuaran su distribución a otros agricultores de la zona. Rápidamente los técnicos percibieron que al cabo de pocas generaciones, muchas cualidades de la variedad desaparecían y la semilla resultante era diferente a la semilla madre creada por el mejorador. Después de algún tiempo y de haber puesto en práctica varios sistemas de multiplicación, se concluyó que era necesario el establecimiento de metodologías apropiadas, supervisadas por técnicos especialistas en el área de semilla, con el objetivo de que la semilla no sufriera deterioro genético de su calidad antes que ésta llegara al productor agrícola. Fue así como se iniciaron los servicios de certificación de semillas hace cerca de un siglo (Martínez, 1982).
Las categorías de semillas se establecen con la finalidad de asegurar que en las distintas multiplicaciones se mantengan las características genéticas y fitosanitarias de los cultivares. La normativa vigente en el Perú (MINAGRI, 2012) establece tres categorías de semillas, las cuales, en cierta forma, coinciden con las normas de semillas establecidas en otras latitudes (MAC, 1986; Oropeza y Mazzani, 1997; Cerda et al., 2000; PNS, 2000; USDA, 2009); estas categorías son las siguientes:

a) Genética, también llamada del mejorador, que representa a la semilla original resultante del proceso de mejoramiento genético, capaz de reproducir la identidad de un cultivar, producida y mantenida bajo el control directo del obtentor, o bajo su dirección o supervisión por otro fítomejorador, en su nombre.

b) Semilla Certificada: es la semilla producida bajo el proceso de certificación por el órgano rector o Autoridad en Semilla. La misma comprende las siguientes categorías:

b.1. Categoría Básica o de Fundación: Es la semilla obtenida a partir de la semilla Genética, producida bajo la supervisión de un fitomejorador o entidad creadora del cultivar y sometida al proceso de certificación, cumpliendo los requisitos establecidos.

b.2. Categoría Registrada: Es aquélla obtenida a partir de la semilla genética, básica o de fundación 
sometida al proceso de certificación y que cumple con los requisitos establecidos para esta categoría de semilla.

b.3. Categoría Certificada: Es aquélla proveniente de semilla genética, de fundación, o de semilla registrada, sometida al proceso de certificación, cumpliendo con los requisitos mínimos establecidos en el reglamento de la especie o grupo de especies.

b.4. Categoría de Semilla Autorizada: Es aquella semilla que posee suficiente identidad y pureza varietal, que ha sido sometida al proceso de certificación y que cumple con los requisitos establecidos para la semilla certificada, excepto en lo que a su procedencia se refiere. Esta categoría de semilla sólo será utilizada en caso de escasez de semillas, previa autorización de la Autoridad en Semillas.

c) Semilla No Certificada: Se entiende por esta categoría de semilla, cualquier semilla que se ofrezca a la venta y que no cumple con los requisitos indicados para la semilla certificada, debido a no haber sido sometida a dicho proceso. Sin embrago, esta semilla deberá rotularse y reunir los requisitos mínimos de calidad establecidos en el reglamento específico de semillas por cultivo y demás disposiciones complementarias. Debido a que durante su producción esta semilla no es sometida a los controles oficiales, la garantía de calidad es responsabilidad de su productor.

Certificación de Semillas: Se entiende por certificación de semilla, el proceso mediante el cual se verifica la identidad, la producción, el acondicionamiento y la calidad de las semillas, de conformidad con lo establecido en la Ley, cuyo propósito es asegurar a los usuarios de semillas, su pureza e identidad genética, así como adecuados niveles de calidad física, fisiológica y sanitaria (El Peruano, 2008).

De acuerdo al Decreto Supremo $N^{\circ} 0026$ 2008-AG (E1 Peruano, 2008), que establece los lineamientos para la producción y comercialización de semilla certificada en el Perú, el sometimiento al proceso de certificación de semillas es voluntario, siendo uno de los requisitos para acceder al proceso de certificación, que los cultivares a ser multiplicados deben estar inscritos en el Registro de Cultivares Comerciales, con excepción de los cultivares destinados exclusivamente a la producción de semillas para la exportación, en cuyo caso, si el país importador requiere el sometimiento al proceso de certificación de semillas, dicho proceso se ejecuta en forma directa, obviando el requisito de inscripción en el Registro de Cultivares Comerciales.

La certificación de semillas es competencia de la Autoridad en Semillas, pudiendo ser ejecutada a través de personas naturales o jurídicas, de los 
público y privado, incluyendo a los productores de semillas, Comités Regionales Departamentales de Semillas, interesados y debidamente calificados, previa delegación o autorización. Las personas delegadas o autorizadas son responsables por la calidad de las semillas que certifican (El Peruano, 2008).

\section{Requisitos exigidos por la Autoridad de Semillas para la producción de semilla de maíz amiláceo de calidad}

En virtud de la importancia que tiene para la región altoandina del Perú la producción de maíz amiláceo y considerando que la norma para la producción, certificación y comercio de semillas de maíz (INIA, 2009) no fomenta la producción de semilla de maíz amiláceo, el INIA aprobó el reglamento para la producción, certificación y comercio de maíz amiláceo (INIA, 2013). En dicho reglamento se establecen las siguientes normas, que rigen la producción, certificación y comercio de maíz amiláceo, presentadas en las tablas 1 y 2:

\section{Aislamiento \\ Para evitar la contaminación entre los lotes de producción de semilla, es indispensable tener un aislamiento adecuado, debido a que el maíz es una planta alógama, (de polinización cruzada). Este aislamiento se puede lograr en tiempo o espacio. En la tabla 2, sección 2.2.4 se indican los parámetros establecidos por el Reglamento General}

de Semilla para el Perú, el cual estipula 300 m. para las categorías Básica y Registrada y 200 m. para las categorías Certificadas y Autorizadas. Cuando el aislamiento se haga en tiempo, la norma establece no menos de 30 días calendario entre la siembra del lote de producción de semilla y la siembra de cualquier otro lote de maíz. Sin embrago, la FAO (2005) indica que el aislamiento en relación a otros campos de maíz deberá ser de $400 \mathrm{~m}$, cuando el lote esté en la dirección del viento y $200 \mathrm{~m}$ cuando están paralelos. Del mismo modo, el Programa de Maíz del CIMMYT y otros investigadores en el área de mejoramiento genético del cultivo de maíz (The Maize Program, 2005; Hallauer et al., 2010), para la semilla genética recomiendan $300 \mathrm{~m}$ de separación de otros campos de maíz que estén floreciendo al mismo tiempo en que ocurre la floración en los lotes de producción de semilla y $200 \mathrm{~m}$ para los lotes de producción de semilla de fundación, pero enfatizan que mientas más distantes mejor. Adicionalmente es importante señalar que existen otros factores que se deben considerar para determinar distancias que aseguren un aislamiento apropiado, entre los cuales figuran la experiencia de campo, materiales de madurez diferencial, el conocimiento que se tenga de la dirección prevaleciente del viento en el momento de la floración y la presencia de barreras naturales o artificiales, entre otros. 
Tabla 1. Normas para la producción de semilla de maíz amiláceo.

GENERALIDADES

1.1. Nombre Común Maíz amiláceo: incluye las razas morocho, chullpi y confite.

1.2. Nombre científico Zea mays L.

Clase Genética

Clase Certificada

1.3. Clases y categorías de semillas
-. Categoría Básica o de Fundación

-. Categoría Registrada

-. Categoría Certificada

-. Categoría Autorizada

Clase no Certificada

1.4. Registros de Cultivares Comerciales.

1.4.1. Ensayos de Adaptación y Eficiencia.

1.4.2. Ensayos de Identificación

\subsubsection{Ejecutor de Ensayo}

Mínimo en dos (02) campañas agrícolas normales y como mínimo en tres (03) localidades diferentes, en donde esté prevista su producción y comercialización.

Mínimo un (01) ensayo por campaña, en dos (02) campañas agrícolas consecutivas.

Instituto Nacional de Innovación Agraria (INIA), Investigadores o Centros de Investigación en Semillas, Inscritos en el Registro Oficial de la Autoridad en Semillas.

1.4.4. Cantidad de Semillas. Suficiente para la instalación de los ensayos.

Los interesados deben presentar copia de la licencia

1.4.5. Ensayos de fitosanitaria de internación del lote de semilla utilizado. Se Cultivares Obtenidos deberácumplir con loindicado en el artículo $65^{\circ}$ del en el Extranjero. $\quad$ Reglamento General de la Ley General de Semillas (D.S. 0006-2012 AG).

Respecto al aislamiento en tiempo, el Programa de Maíz del CIMMYT (The Maize Program, 2005) señala que la siembra de los lotes de producción de semilla debe hacerse ya sea o muy temprano o suficientemente más tarde que otros lotes de maíz vecinos, de modo de asegurar que la emergencia de los estigmas y la liberación del polen se haya completo antes de que emerja la panoja en los materiales vecinos. De cualquier forma, una combinación de fechas de siembra y distancia se pueden utilizar para asegurar un aislamiento apropiado. Por otro lado, Paliwal (2001), señala que los bloques de cruzamiento en la producción de semilla de maíz de las variedades deben tener un aislamiento mínimo de $300 \mathrm{~m}$ respecto de otros lotes de maíz que estén floreciendo al mismo tiempo que los lotes de producción de semilla. 


\section{MATERIALES Y MÉTODOS}

\section{Prácticas agronómicas en la} producción de semillas

Las prácticas agronómicas implementadas en la producción de semilla básicamente son las mismas utilizadas en la producción de grano, excepto por la utilización del riego, la cual se considera una práctica rutinaria en la producción de semilla. No obstante, en esta actividad agrícola el manejo agronómico debe hacerse estrictamente apegado a las exigencias del cultivo, cuidando que las labores se hagan con alta precisión, ya que el mismo no sólo influye en la productividad del genotipo multiplicado, sino que además es determinante de la calidad de la semilla producida.

Las deficiencias nutricionales, déficit o excesos de humedad que se presenten dos semanas antes o después de la floración son altamente perjudiciales, porque afectan directamente el potencial de producción de los genotipos, pero los estreses que se presenten después que ocurra la fecundación del óvulo, durante el proceso de llenado, también son altamente perjudiciales, porque influyen negativamente sobre la calidad de la semilla, puesto que se afecta de manera directa el desarrollo de las estructuras embrionarias de la semilla, responsables de dar origen al nuevo individuo, como lo son la radícula y hojas embrionarias.

En consecuencia, la producción de semilla debe realizarse con una buena preparación de los suelos, complementada con una adecuada fertilización, en función de los requerimientos del genotipo producido y tomando en cuenta los aportes que tenga el suelo, aplicando el riego en la cantidad y frecuencia requerida por la planta en función de la etapa de desarrollo, manteniendo las siembras bajo un estricto control fitosanitario, que permitan el desarrollo del cultivo libre de las presiones de los insectos plagas, malezas nocivas y de patógenos responsables de las principales enfermedades que afectan al cultivo.

Tabla 2. Normas para la certificación de semilla básica, Registrada, Certificada y Autorizada de maíz amiláceo.

\section{GENERALIDADES}

\section{DESCRIPCIÓN}

2.1. Verificación Preliminar

\subsubsection{Presentación de la} Solicitud de Inscripción.

Máximo hasta 30 días después de la siembra.

a. Incumplimiento del plazo de presentación de la solicitud de inscripción del campo de multiplicación.

\subsubsection{Causales de Rechazo} Solicitud de Inscripción

b. Incumplimiento de presentar los requisitos contemplados en el artículo 17 del Reglamento Técnico de Certificación de Semillas (D.S. N 024-2005 AG.) 


\begin{tabular}{|c|c|}
\hline & $\begin{array}{l}\text { c. Incumplimiento del área mínima del campo de } \\
\text { multiplicación. } \\
\text { d. Incumplimiento de la rotación del campo de multiplicaci- } \\
\text { ón }\end{array}$ \\
\hline \multicolumn{2}{|l|}{$\begin{array}{l}\text { 2.2. Inspecciones de } \\
\text { Campo. }\end{array}$} \\
\hline $\begin{array}{l}\text { 2.2.1. } \mathrm{N}^{\circ} \text { de } \\
\text { Inspecciones de } \\
\text { Campo. }\end{array}$ & $\begin{array}{l}\text { Se realizarán tres }(03) \text { inspecciones de campo: } \\
\text { a. Primera: Antes de la floración masculina, con el propósito } \\
\text { de verificar la rotación, distancia de aislamiento, pureza } \\
\text { varietal y estado culturaldel campo de multiplicación, así } \\
\text { como también, verificar el área y la cantidad de semilla } \\
\text { utilizada. } \\
\text { b. Segunda: después de la floración, para comprobar la pureza } \\
\text { varietal de las plantas. } \\
\text { c. Tercera: Durante la cosecha, para comprobar la pureza } \\
\text { varietal de los granos. } \\
\text { Es responsabilidad del productor la eliminación de plantas } \\
\text { voluntarias, atípicas y enfermas. } \\
\text { Los campos de multiplicación deberán estar ubicados, } \\
\text { preferentemente, cerca de las vías de comunicación, a fin de } \\
\text { permitir las inspecciones en forma regular. }\end{array}$ \\
\hline $\begin{array}{l}\text { 2.2.2. Tamaño mínimo } \\
\text { de campos (has) }\end{array}$ & $\begin{array}{l}\text { Para las categorías de semillas Certificadas y Autorizadas se } \\
\text { exige un mínimo de } 0.5 \text { ha. Para las categorías Básica y } \\
\text { Registrada no se tiene un mínimo establecido. } \\
\text { Cuando los campos de multiplicación tengan limitaciones de } \\
\text { accesibilidad o estén ubicados en lugares muy distantes de las } \\
\text { vías de comunicación, el área total de las solicitudes de } \\
\text { certificación deberán ser como mínimo para } 1 \text { ha, quedando a } \\
\text { criterio del organismo certificador aceptar áreas menores. }\end{array}$ \\
\hline 2.2.3. Rotación & $\begin{array}{l}\text { Los campos de producción no deben haber sido sembrados con } \\
\text { maíz en la campaña anterior. } \\
\text { En el caso de cultivares de polinización abierta se podrán } \\
\text { aceptar solicitudes de inscripción de campos que el ciclo } \\
\text { anterior hayan estado sembrados con el mismo cultivar. En el } \\
\text { caso de tratarse de un cultivar diferente, se aceptará si el } \\
\text { productor de semilla ha realizado labores culturales para } \\
\text { provocar la germinación y eliminación de todas las semillas } \\
\text { remanentes en el campo, lo cual será verificado por el } \\
\text { organismo Certificador. }\end{array}$ \\
\hline $\begin{array}{l}\text { 2.2.4. Aislamiento del } \\
\text { campo respecto a otros } \\
\text { c a m o s d e maíz } \\
\text { (mínimo en } \mathrm{m} . \text { ) }\end{array}$ & $\begin{array}{l}300 \text { m. para las categorías Básica y Registrada y } 200 \mathrm{~m} \text {. para las } \\
\text { categorías Certificadas y Autorizadas. } \\
\text { Se puede permitir también aislamiento por tiempo, con } \\
\text { siembras diferencias de no menos de } 30 \text { días calendario. El } \\
\text { organismo de certificación verifica que, no obstante el }\end{array}$ \\
\hline
\end{tabular}




\begin{tabular}{|c|c|}
\hline & $\begin{array}{l}\text { aislamiento por tiempo en la siembra, los períodos de floración } \\
\text { no coincidan. Se podrán aceptar siembras con un período menor } \\
\text { de } 30 \text { días, si se verifica que el campo contaminante no emite } \\
\text { polen durante el período de producción de estigmas receptivos } \\
\text { del campo de multiplicación de semillas. }\end{array}$ \\
\hline $\begin{array}{l}\text { 2.2.5. Plantas fuera de } \\
\text { tipo de Cultivares de } \\
\text { Polinización Abierta } \\
\text { (Número máximo) }\end{array}$ & $\begin{array}{l}\text { Para los campos de producción de semilla básica y Registrada } \\
\text { se acepta hasta un máximo de } 5 / 1000 \text { plantas, mientras que para } \\
\text { la producción de semilla Certificada y Autorizada, los campos } \\
\text { de producción podrán tener un máximo de } 10 / 1000 \text { plantas. }\end{array}$ \\
\hline 2.2.6. Malezas & $\begin{array}{l}\text { Los campos de multiplicación deben estar libres de malezas } \\
\text { durante todo el período de producción. En caso el inspector } \\
\text { observe la presencia de malezas, dispondrá su inmediata } \\
\text { eliminación. }\end{array}$ \\
\hline $\begin{array}{l}\text { 2.2.7. Causas de } \\
\text { Rechazo del Campo } \\
\text { de Multiplicación }\end{array}$ & $\begin{array}{l}\text { a. Incumplimiento del área mínima del campo de } \\
\text { multiplicación. } \\
\text { b. Incumplimiento de la rotación del campo de multiplicación. } \\
\text { c. Incumplimiento de las tolerancia establecidas en los } \\
\text { numerales } 2.2 .4 \text { al } 2.2 .6 \text {. } \\
\text { d. Incumplimiento de las recomendaciones del inspector, } \\
\text { respecto a los controles de malezas yotras. } \\
\text { e. Presentar información falsa sobe el total de producción del } \\
\text { campo de multiplicación. } \\
\text { Utilizar el informe de inspección de campo con fines de } \\
\text { comercialización de la semilla. }\end{array}$ \\
\hline $\begin{array}{l}2.3 \text { Declaración de la } \\
\text { cosecha. }\end{array}$ & $\begin{array}{l}\text { Se permitirá declarar un peso estimado del total de la } \\
\text { producción del campo de multiplicación de semillas, en } \\
\text { variedades con con contenido de humedad de mazorcas mayor a } \\
25 \% \text { (madurez fisiológica). }\end{array}$ \\
\hline 2.4 Acondicionamiento & \\
\hline \multirow[t]{2}{*}{$\begin{array}{l}\text { 2.4.1. Planta de } \\
\text { Acondicionamiento } \\
\text { Registrada }\end{array}$} & $\begin{array}{l}\text { El Organismo Certificador, podrá admitir el acondicionamiento } \\
\text { manual o artesanal, siempre que dicha operación contemple las } \\
\text { medidas necesarias para que los lotes de semillas conserven el } \\
\text { orden, la separación e identificación, de manera que se eviten } \\
\text { mezclas y cambios de lotes que afecten la calidad e identidad de } \\
\text { la semilla y se facilite la inspección de certificación. } \\
\text { Equipamiento e infraestructura mínima: } \\
\checkmark \text { Área de secado } \\
\quad \checkmark \text { Zarandas } \\
\quad \checkmark \text { Balanzas } \\
\text { Área de almacenamiento }\end{array}$ \\
\hline & $\begin{array}{l}\text { En la etapa de acondicionamiento, el productor de semilla debe } \\
\text { cumplir las siguientes disposiciones: }\end{array}$ \\
\hline
\end{tabular}




\begin{tabular}{|c|c|}
\hline $\begin{array}{l}\text { 2.4.2. Disposiciones } \\
\text { para el } \\
\text { Acondicionamiento }\end{array}$ & $\begin{array}{l}\text { a. En el caso de descalificación parcial del campo de } \\
\text { multiplicación, se cosechará sólola parte aprobada. } \\
\text { b. Deberá hacerse una completa y cuidadosa selección de } \\
\text { mazorcas antes del secado. Si fuera necesario, se procederá } \\
\text { a una reselección previo al desgrane. Las mazorcas fuera de } \\
\text { tipo o dudosas, así como las que presenten deficiente estado } \\
\text { sanitario, deberán ser eliminadas. }\end{array}$ \\
\hline $\begin{array}{l}\text { 2.4.3. Parámetros de } \\
\text { inspección }\end{array}$ & $\begin{array}{l}\text { a. El área de acondicionamiento recibe la cosecha } \\
\text { debidamente identificada y los lotes se almacenan en áreas } \\
\text { con adecuada ventilación, limpieza y desinfección. } \\
\text { b. Para el acondicionamiento debe contemplarse la limpieza de } \\
\text { residuos en los equipos de clasificación y transporte. Cuando } \\
\text { se trate del mismo cultivar, es recomendable que el proceso } \\
\text { se inicie con la categoría Básica, seguida de la Registrada y } \\
\text { concluir con la Certificada. } \\
\text { c. Envasado: Deben utilizarse envases nuevos y limpios. El } \\
\text { material de los envases no debe afectar la calidad de las } \\
\text { semillas. } \\
\text { d. Almacenamiento: La semilla almacenada deberá estar } \\
\text { dispuesta sobre tarimas que permita el acceso del inspector } \\
\text { a todos los envases. }\end{array}$ \\
\hline $\begin{array}{l}\text { 2..4.4. Tolerancia de } \\
\text { mazorcas fuera de tipo } \\
\text { (Máximo) }\end{array}$ & $\begin{array}{l}\text { Para las categorías Básica y Registrada, la tolerancia máxima } \\
\text { de mazorcas fuera de tipo es de } 1 \text { por cada mil mazorcas, } \\
\text { mientras que en las categorías Certificad y Autorizada, se } \\
\text { aceptan hasta } 2 \text { mazorcas fuera de tipo por cada } 1000 \text { mazorcas } \\
\text { producidas. } \\
\text { En el caso de excederse los límites establecidos, podrá } \\
\text { hacerse una nueva inspección a solicitud del productor, } \\
\text { después de una cuidadosa selección del lote. }\end{array}$ \\
\hline $\begin{array}{l}\text { 2.4.5. Causales de } \\
\text { Rechazo de Lotes de } \\
\text { Semillas }\end{array}$ & $\begin{array}{l}\text { a. No brindar las facilidades para que el inspector ejecute una } \\
\text { evaluación adecuada. } \\
\text { b. El desgrane o trilla antes de la inspección, descalificará el lote } \\
\text { de semilla para su certificación. } \\
\text { c. El incumplimiento de las tolerancias establecidas para las } \\
\text { mazorcas fuera de tipo. } \\
\text { d. El acondicionamiento de semilla sin conservar el orden, } \\
\text { separación e identificación. } \\
\text { e. El acondicionamiento de semilla sin considerar el peso } \\
\text { máximo del lote de semillas establecido en las reglas ISTA. } \\
\text { f. Presencia de plagas o el efecto de factores adversos que } \\
\text { comprometan la calidad de las semillas oque no permitan } \\
\text { efectuar una correcta evaluación del lote de semillas. } \\
\text { g. Incumplimiento de las tolerancias para el análisis de semillas } \\
\text { (numeral 2.5), siempre que no exista la posibilidad de } \\
\text { reacondicionar el lote de semillas, para cumplir con dichas } \\
\text { exigencias. } \\
\text { h. Utilizar el informe de acondicionamiento con fines de } \\
\text { comercialización. }\end{array}$ \\
\hline
\end{tabular}




\begin{tabular}{|c|c|c|c|c|}
\hline & \multicolumn{4}{|c|}{$\begin{array}{l}\text { i. Almacenamiento en condiciones evidentemente } \\
\text { perjudiciales para el lote acondicionado. }\end{array}$} \\
\hline $\begin{array}{l}\text { 2.5. Análisis de la } \\
\text { Semilla }\end{array}$ & Básica & Registrada & Certificada & Autorizada \\
\hline $\begin{array}{l}\text { 2.5.1. Peso máximo de } \\
\text { Lote }\end{array}$ & \multicolumn{4}{|c|}{ De acuerdo a las reglas ISTA } \\
\hline $\begin{array}{l}\text { 2.5.2. Peso mínimo de } \\
\text { Muestra }\end{array}$ & \multicolumn{4}{|c|}{ De acuerdo a las reglas ISTA } \\
\hline $\begin{array}{l}\text { 2.5.3. Semilla Pura }(\% \\
\text { mínimo) }\end{array}$ & 98 & 98 & 98 & 98 \\
\hline $\begin{array}{l}\text { 2.5.4. Materia Inerte } \\
\text { (\% máximo) }\end{array}$ & 2 & 2 & 2 & 2 \\
\hline $\begin{array}{l}\text { 2.5.5. Otras Semillas } \\
(\% \text { máximo })\end{array}$ & 0 & 0 & 0 & 0 \\
\hline $\begin{array}{l}\text { 2.5.6. Germinación } \\
\text { (\% mínimo) }\end{array}$ & 85 & 85 & 85 & 85 \\
\hline \multicolumn{5}{|l|}{ 2.6. Envasado y Etiquetado } \\
\hline $\begin{array}{l}\text { 2.6.1. Vigencia de la } \\
\text { Etiqueta de } \\
\text { Certificación. }\end{array}$ & \multicolumn{4}{|c|}{$\begin{array}{l}\text { - Nueve (09) meses a partir de su etiquetado oficial y en } \\
\text { condiciones apropiadas para su conservación. } \\
\text { - Después de este período, el productor de semillas solicitará } \\
\text { al Organismo Certificador el re-etiquetado de los lotes, } \\
\text { previa inspección de los mismos, para la toma de muestras y } \\
\text { análisis de calidad. }\end{array}$} \\
\hline
\end{tabular}

\section{RESULTADOS}

Inspecciones en el proceso de producción de semilla: La supervisión del proceso de producción de semilla es una actividad permanente, la cual es realizada tanto por personal técnico de la empresa o entidad productora, como por funcionarios oficiales de la Autoridad en Semillas (El Peruano, 2008). Las inspecciones son ejecutadas durante varias fases del proceso de producción en el campo, en las plantas procesadoras y en los depósitos de almacenamiento de semilla, con la finalidad de velar por que se cumplan los requisitos establecidos en las normas de certificación, realizar las observaciones y verificaciones pertinentes y orientar a los productores cooperadores, para garantizar la obtención de semilla de alta calidad (Oropeza et al., 2000).

\section{Producción de semilla híbrida}

La producción de semilla híbrida de maíz es posible realizarla de dos maneras diferentes: 
una, por medio del uso de la androesterilidad génica citoplasmática, que permite el uso de un progenitor femenino, que produce polen estéril, lo cual garantiza que la fertilización de las hileras de plantas hembras sea realizada por el polen proveniente del progenitor masculino, y la otra, a través del uso de parentales femeninos (líneas o híbridos) que producen polen fértil, por lo cual requieren ser despanojados (emasculadas) en forma manual o mecánica, para asegurar que la polinización sea efectuada con polen proveniente del progenitor masculino
(Wych, 1988). En este caso, el despanojado se inicia tan pronto emergen las primeras panojas en las hileras de plantas madres y se extiende durante alrededor de unas dos semanas.

Durante el lapso de tiempo que dure el proceso de despanojado, se efectúa el recorrido diario del total de hileras hembras de cada lote de producción, utilizando cuadrillas de obreros debidamente entrenados en las labores del despanojado, quienes aseguran la eliminación de todas las panojas en las hileras madres antes de que ocurra la antesis (figura 1).
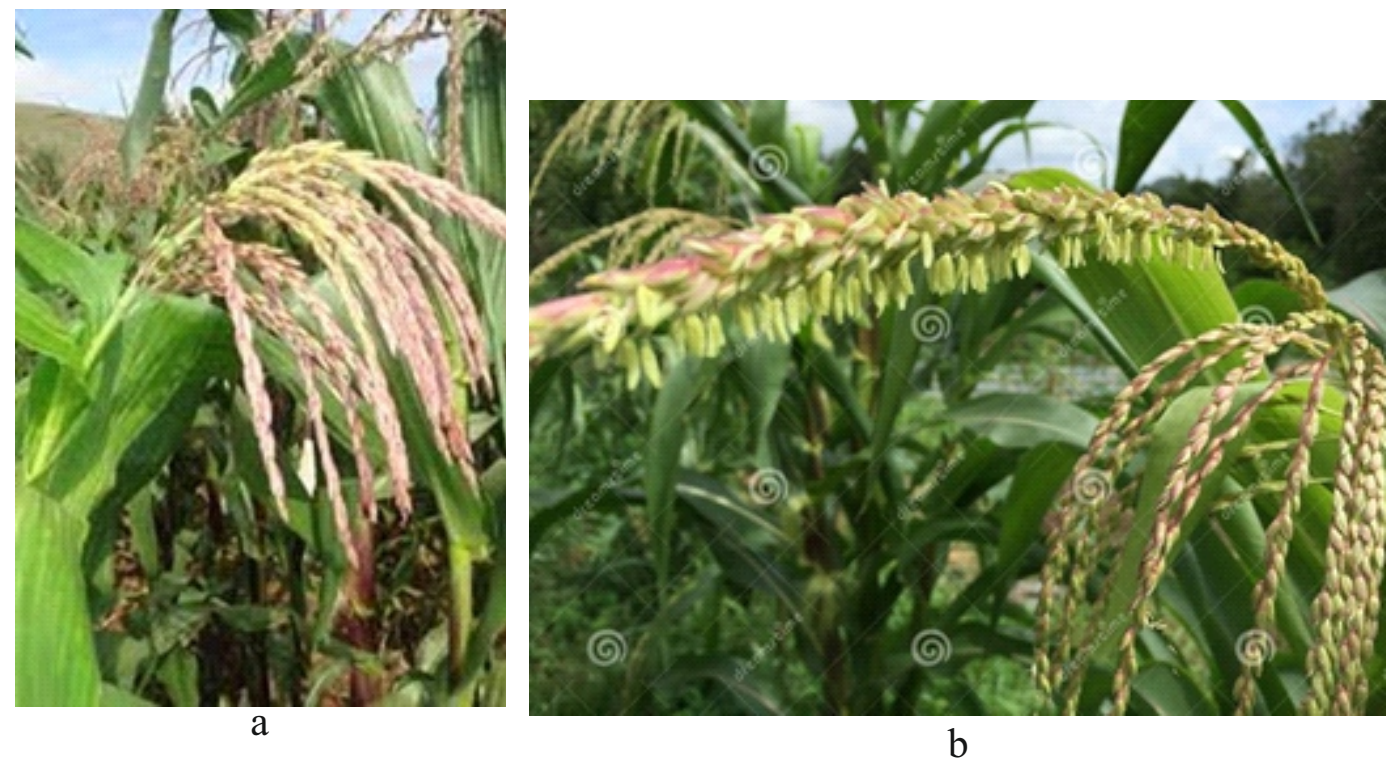

Figura 1. Momento oportuno de realizar el despanojado, antes de que ocurra la antesis (a). En la Figura 1 b ya ocurrió la antesis y por ende habrá contaminación de la semilla híbrida producida.

La práctica del despanojado consiste en la remoción física de la panoja de todas las plantas hembras, antes de que derramen polen, de modo que se asegure que todos los granos en las mazorcas de las hileras de plantas femeninas hayan sido polinizados con polen de los progenitores masculinos, garantizando así una adecuada calidad de semilla del híbrido. El despanojado manual se efectúa tirando hacia arriba de la parte inferior del pedúnculo de la panoja. Cuando la densidad de siembra en las hileras hembras es muy alta y los espaciamientos 
entre hileras son muy bajos, la eficiencia del despanojado puede ser baja (Granados, 2001). El despanojado es una actividad de corta duración pero muy intensiva que no se puede interrumpir en caso de mal tiempo o de días festivos, debido a que afecta directamente la calidad de la producción de la semilla del híbrido. En cualquiera de las inspecciones de despanojado, cuando las plantas femeninas tienen más de $5 \%$ de estambres receptivos, los surcos masculinos no deberían presentar más de $0,2 \%$ de plantas fuera de tipo derramando polen y las plantas femeninas no deberían tener más de $1 \%$ de panojas polinizando (Agrawal et al., 1998). Al final del proceso de despanojado, todas las hileras hembras se muestran sin espigas y sólo presentaran la inflorescencia masculina las hileras machos.

La eficiencia de la producción de semillas es importante para el éxito de cualquier programa de semillas. De acuerdo a Paliwal (2001), es deseable organizar las diferentes etapas de la producción de semillas en la región de interés, a fin de evitar problemas de deriva genética y disminuir los costos de transporte. Por otro lado, un elemento importante es la tasa de multiplicación de la semilla, desde la semilla genética hasta la semilla certificada, de manera que se produzcan las cantidades adecuadas de semillas en áreas relativamente pequeñas, que permitan una supervisión e inspección de los campos de producción más eficiente, manteniendo bajos los costos (Paliwal,
2001).

El manejo de los lotes de producción de semillas también debe ser el mejor posible, con el aporte oportuno de todos los insumos necesarios. Se recomienda utilizar poblaciones de plantas en las parcelas de producción de semillas uniformes y adecuadas al cultivar; el tamaño de la semilla será mayor si la densidad de las plantas es relativamente baja y viceversa.

Será siempre conveniente conservar existencias de semillas de categoría genética y fundación de reserva, ya que en caso del fracaso inesperado de un cultivo, el programa de producción no se interrumpa y se pueda mantener un abastecimiento regular de semillas a los agricultores (Paliwal, 2001).

La producción de semillas es un sistema continuo, desde la producción de semillas genética hasta las semillas certificadas, por lo que se requiere hacer una planificación cuidadosa para evitar déficit o excesos de semillas y mantener un abastecimiento regular. Normalmente, la planificación se hace estimando la cantidad de semilla certificada requerida para un área determinada y a partir de allí se asegura su producción por medio de una adecuada producción de semillas de las categorías superiores.

En las Tablas 3 y 4 se muestran las áreas y diferentes categorías de semilla requeridas para la producción de 100.000 ha de maíz, con una variedad de libre polinización y con un híbrido simple, 
respectivamente. En el caso de la producción de semilla del híbrido simple (tabla 4), se consideró una relación hembra:macho 4:1, o sea, cuatro hileras del progenitor femenino por una hilera de del masculino. Dowswell et al, (1996) prepararon una guía similar para la planificación de la producción de semillas de híbridos dobles de maíz, para la siembra comercial de 200.000 hectáreas, que representa un caso más complejo, ya que involucra la planificación de los requerimientos y producción de cuatro líneas endocriadas y dos cruzamientos simples, los que son usados posteriormente para la producción del híbrido doble.

La producción de semilla de híbridos es compleja y costosa y los productores de semilla deben aprender diseños complicados para la siembra; la siembra de surcos femeninos y masculinos se debe hacer en tal forma que se sincronice la emisión de estambres de los primeros con el derrame del polen de las plantas masculinas. También se deben establecer normas más exigentes, entresacar plantas y despanojar y tener sumo cuidado en la cosecha.

El mayor costo de las semillas de los parentales contribuye al mayor costo de las semillas del híbrido, pero sin embargo, es provechoso para los agricultores pagar por ese mayor precio de la semilla si las ganancias esperadas por su uso son adecuadas en términos de productividad y económicos. CIMMYT (1987, 1994), López-Pereira y Filippello (1995) y Dowswell et al, (1996) han discutido varios aspectos de la economía de la producción de semillas en los países en vías de desarrollo.

Tabla 3. Planificación para la producción de diferentes categorías de semillas, destinadas a la siembra de cien mil (100000) hectáreas de maíz comercial de una variedad de libre polinización.

\begin{tabular}{lcccc}
\hline \multicolumn{1}{c}{ Actividad } & $\begin{array}{c}\text { Rendimiento } \\
\text { esperado }\end{array}$ & Área & $\begin{array}{c}\text { Densidad de } \\
\text { siembra }\end{array}$ & $\begin{array}{c}\text { Semilla a } \\
\text { necesitar }\end{array}$ \\
\hline & $(\mathrm{tn} / \mathrm{ha})$ & $(\mathrm{ha})$ & $\mathrm{kg}$ & $\mathrm{kg}$ \\
Cultivo comercial & - & 100000 & 20 & 2000000 \\
Semilla certificada & 3.500 & 571.429 & 15 & 8571.435 \\
Semilla fundación & 2.500 & $6.858^{*}$ & 15 & 102.870 \\
Semilla breeder & 1.500 & $0.069^{*}$ & 10 & 0.690 \\
\hline
\end{tabular}

* Esta área es el doble del área real necesaria, para producir una cantidad adicional de semillas de reserva.

Fuente: adaptado de CIMMYT (1984); Dowswell et al. (1996). Cálculos propios. 
Tabla 4. Planificación para la producción de diferentes categorías de semillas, destinadas a la siembra de cien mil (100000) hectáreas de maíz comercial de un híbrido simple.

\begin{tabular}{lccccc}
\hline \multicolumn{1}{c}{ Actividad } & $\begin{array}{c}\text { Rendimiento } \\
\text { esperado } \\
\text { (tn/ha) }\end{array}$ & $\begin{array}{c}\text { Área } \\
\text { (ha) }\end{array}$ & $\begin{array}{c}\text { Densidad de } \\
\text { siembra } \\
\text { (kg) }\end{array}$ & $\begin{array}{c}\text { Semilla a } \\
\text { necesitar (PF) } \\
(\mathbf{k g})\end{array}$ & $\begin{array}{c}\text { Semilla a } \\
\text { necesitar (PM) } \\
\mathbf{( k g )}\end{array}$ \\
\hline Cultivo comercial & - & 100000 & 20 & \multicolumn{2}{c}{2000000} \\
Semilla certificada & 2.000 & 1000 & 15 & 15000 & 5000 \\
$\begin{array}{l}\text { Semilla fundación } \\
\text { Semilla breeder }\end{array}$ & 1.500 & $20^{*}$ & 15 & 300 & 75 \\
\begin{tabular}{l} 
(Genética) \\
\hline
\end{tabular} & 1.000 & $0.60^{*}$ & 10 & 6.00 & 1.50 \\
\hline
\end{tabular}

Esta área es el doble del área real necesaria, para producir una cantidad adicional de semillas de reserva.

Fuente: adaptado de CIMMYT (1984); Dowswell et al. (1996). Cálculos propios.

PF y PM significan progenitor femenino y progenitor masculino, respectivamente.

\section{Cosecha y acondicionamiento de semilla}

La cosecha puede hacerse en forma manual o mecanizada, mediante el uso de cosechadoras de mazorca. La misma se puede iniciar desde cuando la semilla alcanza 30\% de humedad. En el caso de la semilla híbrida, se recomienda cosechar el macho polinizador en la etapa de maíz tierno (Choclo), para evitar contaminaciones en la cosecha de las hileras hembras, al confundir las mazorcas del parental masculino con las mazorcas valiosas de las plantas femeninas.

Una vez practicada la cosecha, las mazorcas son trasladadas a los galpones de las chacras, para la eliminación de mazorcas y granos fuera de tipo o con características indeseables para su uso como semilla. Según Oropeza et al. (2000), la presencia de más del $1 \%$ de 166 Rev. Tayacaja 2(2): 150 - 176, 2019 ISSN:2617-9156 mazorcas atípicas en una inspección, obligará a la reclasificación de las mazorcas de todo el lote en cuestión.

Es importante mencionar que la apariencia de la semilla no siempre representa un aspecto de calidad, pero afecta el mercadeo y la promoción, debido a que los productores no pueden apreciar en la semilla las bondades genéticas del cultivar que están comprando, tales como la presencia de genes de resistencia a enfermedades importantes o de adaptación a condiciones específicas, etc, pero si es capaz de apreciar si una semilla está bien acondicionada. No obstante, Johnson (1980) refiere que aunque el acondicionamiento es el medio principal de remoción de contaminantes de la semilla, también puede constituir una fuente importante de contaminación. Por consiguiente, el acondicionamiento debe 
realizarse evitando contaminar la semilla con semillas de otros cultivares procesados previamente y sin causarle daños mecánicos.

Durante el acondicionamiento la calidad de la semilla puede ser mejorada de dos maneras, mediante la eliminación de semillas contaminantes de otros cultivos, malezas y materia inerte y mediante la eliminación de las semillas de baja calidad. El objetivo principal del procesamiento de la semilla es obtener el máximo porcentaje de semilla pura con el máximo potencial de germinación (Copeland, 1976). En consecuencia, la planta beneficiadora es una de las inversiones de capital más grandes en un programa de semillas, convirtiéndose en un activo de alto valor en la operación cuando está bien equipada y administrada, o en un pasivo oneroso cuando ocurre lo contrario (Johnson, 1980).

Toda la semilla que se utiliza para la siembra requiere de algún tipo de acondicionamiento y a medida que se mecanizan los sistemas de producción y cosecha, el acondicionamiento de la semilla se hace más importante.

Los procesos de cosecha y acondicionamiento de la semilla de maíz, incluyendo las etapas de secado, limpieza, separación por tamaño, tratamiento, envasado y almacenamiento hasta su distribución a los agricultores, han sido descriptos en diversas publicaciones (Copeland, 1976; Johnson, 1980; Dowsewll et al., 1996; Agrawal et al.,
1997; Oropeza et al., 2000). La semilla de maíz está técnicamente apta para ser cosechada cuando se ha formado la capa negra en el punto de inserción en el raquis (tusa o coronta) y la semilla ha alcanzado la madurez fisiológica, momento en el cual ha alcanzado su máxima calidad. A partir de entonces, a medida que pasa el tiempo y es sometida a varias fuerzas externas, y su calidad comienza a declinar (Paliwal, 2001).

El contenido de humedad de la semilla en el momento de la madurez fisiológica está por encima del $30 \%$ y en algunos genotipos y condiciones ambientales puede llegar a 38\% (Paliwal, 2001). En muchos ambientes tropicales la semilla no debe ser cosechada con tales niveles de humedad y se considera seguro dejarla secar en la planta hasta cerca de $25 \%$.

Gran parte del maíz para semilla en los países tropicales es cosechado manualmente, al contrario de lo que ocurre en las zonas templadas en los países industrializados donde es cosechado mecánicamente. En la práctica, la cosecha manual es mejor que la mecánica, porque permite al productor de semillas revisar la condición de sanidad de las mazorcas, sin embargo, tiene la desventaja de requerir gran cantidad de mano de obra, recurso altamente escaso en la mayoría de los países tropicales, siendo este problema más pronunciado en ciertos países que mantienen elevados niveles de subvenciones a la población o en donde 
existen ciertas actividades, tales como la actividad minera, que demandan gran cantidad de mano de obra y en donde los trabajadores pueden recibir mejores remuneraciones que la permitida en la actividad agrícola.

\section{Secado}

De acuerdo a Oropeza et al. (2000), dentro de las etapas de acondicionamiento de la semilla, posiblemente el secado sea la más importante, debido a que a través de este proceso se disminuyen los contenidos de humedad de la semilla hasta niveles considerados satisfactorios para su conservación. Es recomendable llevar la humedad de la semilla por debajo del 15\% para su desgranado y procesamiento.

Existen diferentes formas de realizar esta labor, dependiendo del volumen de mazorcas a secar y de las facilidades disponibles. El sistema más económico es el secado al sol, extendiendo las mazorcas en una capa fina, removiéndolas frecuentemente, pero el mismo sólo es eficiente para cantidades relativamente pequeñas. En el caso de la Sierra Peruana, la mayoría de los productores utilizan este sistema de secado al sol, aprovechando que para la época de la cosecha es muy poco probable la ocurrencia de precipitaciones. Para llevar a cabo esta labor, los agricultores cubren la superficie del suelo con gramíneas secas o restos de cosecha, sobre lo cual colocan las mazorcas de manera ordenada y casi en forma vertical. Los agricultores de mayores recursos, pueden utilizar mantas extensas sobre las cuales colocan las mazorcas, cubriéndolas con otra manta en caso de que ocurran precipitaciones. Sin embrago, el sistema más utilizado es el pasaje de aire forzado o secado artificial, ya sea con el aire a temperatura ambiente o con aire caliente. Es el sistema más eficiente, porque da menos oportunidades a las infecciones y al daño de las semillas. Normalmente, la labor del secado se realiza en dos etapas, un presecado en mazorcas, pasando una corriente de aire relativamente seco a través de la masa de mazorcas, a temperaturas de 40 a $43^{\circ} \mathrm{C}$, llevando la semilla entre $16 \%$ y $17 \%$ de humedad, en 24 horas aproximadamente, luego de lo cual se practica el desgrane del lote, para el secado final, llevando la semilla a un $12 \%$ de humedad en unas 10 a 12 horas, aproximadamente, utilizando secadoras verticales convencionales para granel (Oropeza et al. 2000).

Un secador consiste en un ventilador que fuerza el pasaje del aire a través de un elemento caliente como un quemador o un calentador y lo empuja hacia las mazorcas en el área de secado. La fuente de energía puede ser diversa, desde el uso de madera, carbón, tusas de maíz, combustible diesel, gas natural, butano o propano y hasta secadores solares.

Es importante que el quemador o el calentador tengan un control termostático de la temperatura para evitar daños a las semillas. Las temperaturas excesivamente altas combinadas con una 
humedad superior al 20\% pueden dañar irreversiblemente el embrión (Paliwal, 2001).

La regla general es que cuanto más alto es el contenido de humedad de la semilla, más baja debe ser la temperatura del aire que llega a las semillas. Cuando las semillas se secan con aire caliente forzado, la pérdida inicial de humedad es alta, desde la capa exterior del endosperma en un gradiente de humedad desde el centro de la semilla hacia la periferia. Si este gradiente es muy grande, lo cual sucede en semillas con un alto contenido de humedad ( $>25 \%$ ), ocurren una serie de estreses internos, que pueden dañar el pericarpio, originando una menor viabilidad y longevidad de la semilla (Paliwal, 2001).

Existen cuadros disponibles para orientar al usuario sobre la temperatura del aire y el sistema de calentamiento, dependiendo de la cantidad de maíz, su humedad y su humedad relativa.

\section{Desgrane}

Es el proceso mediante el cual se separa la semilla del raquis o tusa. Esta labor se realiza luego del proceso de presecado o secado de las mazorcas, utilizando desgranadoras mecánicas. En algunas plantas procesadoras de semillas se utilizan desgranadoras específicas para semillas de maíz, diseñadas con cóncavos perforados y cilindros cónicos ajustables, que giran a velocidades relativamente bajas (entre 400 a $500 \mathrm{rpm}$ ) (Oropeza et al. 2000).
De cualquier forma, inevitablemente resultan semillas partidas, que pueden ser eliminadas durante las etapas de limpieza, pero también se presentan daños mecánicos ocultos, que no afectan la integridad dimensional de la semilla, pero si generan disminución del vigor, debido a un incremento de la tasa de respiración.

\section{Limpieza}

El proceso de limpieza normalmente es realizado en dos etapas, una primera llamada prelimpieza, donde se eliminan las impurezas mayores, tales como restos de tusas o coronta, impurezas provenientes del campo, semillas muy pequeñas anormalmente grandes, así como también residuos muy livianos provenientes de la coronta y/o semillas (Oropeza et al., 2000).

Normalmente los equipos de prelimpieza están diseñados con un mecanismo para aspiración de polvo y partículas livianas y con dos tipos diferentes de cedazos planos; el primero, para retener los contaminantes pequeños, tales como trozos pequeños de semillas, corontas, hojas, etc, (Oropeza et al., 2000).

El trabajo de limpieza viene a complementar el trabajo previo, de prelimpieza, eliminando los restos contaminantes que hayan quedado en la masa de semilla, realizando a la vez, una preclasificación dimensional de la semilla. Oropeza et al. (2000) refiere, que las máquinas limpiadoras en Venezuela son de dos aspiraciones (al ingreso y 
regreso del material) y de 3 a 5 cedazos planos, de inclinación y velocidad variables, las cuales son muy eficientes en las separaciones de los materiales. A través de cedazos con diferentes tipos y dimensiones de perforación, las máquinas de limpieza retienen las semillas consideradas deseables, separando contaminantes grandes o muy pequeños, así como también semillas livianas y otros materiales de baja densidad, que aún hayan quedado de la etapa de prelimpieza. Para el caso de los maíces amiláceos de la sierra peruana y de todos los Andes de Suramérica, este tipo de equipos tendrían que adaptarse a las condiciones del grano estos maíces, los cuales se caracterizan por ser normalmente más grandes y muchos más blandos que el maíz amarillo duro o el blanco duro.

\section{Clasificación por forma y tamaño}

El tamaño es la característica que se puede identificar con más facilidad en un lote de semillas (figura 2). Las diferencias en longitud, ancho y espesor pueden presentarse entre las mismas semillas o entre éstas y sus contaminantes. Por otro lado, las semillas pueden tener diferente ancho y el mismo espesor como ocurre con las semillas chatas de maíz y que se separan con zarandas con perforaciones redondas y también pueden tener diferencias en espesor como sucede con las semillas chatas y redondas (punta de mazorca) de maíz que se separan con zarandas con perforaciones oblongas.

Las diferencias en longitud de las semillas no permiten hacer separaciones con zarandas y tienen que realizarse con equipos de separación por longitud. Los separadores de longitud son de dos tipos: el separador de discos y el separador de cilindros.

Esta etapa es ejecutada mediante el uso de tres equipos en serie con cedazos cilíndricos giratorios; el primero, de perforaciones transversales alargadas en el fondo de delgados canales, que retiene las semillas de mayor grosor, tipo redondo, dejando pasar las restantes, achatadas o planas, que sucesivamente se llevan a cedazos con perforaciones circulares; el segundo, retiene las semillas achatadas o planas de mayor ancho (plano grande) y el tercero, retiene las semillas de ancho intermedio (plano mediano), dejando pasar las semillas plano pequeño (Oropeza et al., 2000). Las semillas de cada forma y dimensión son recogidas por separado, identificándose cada tipo al momento del envasado. 

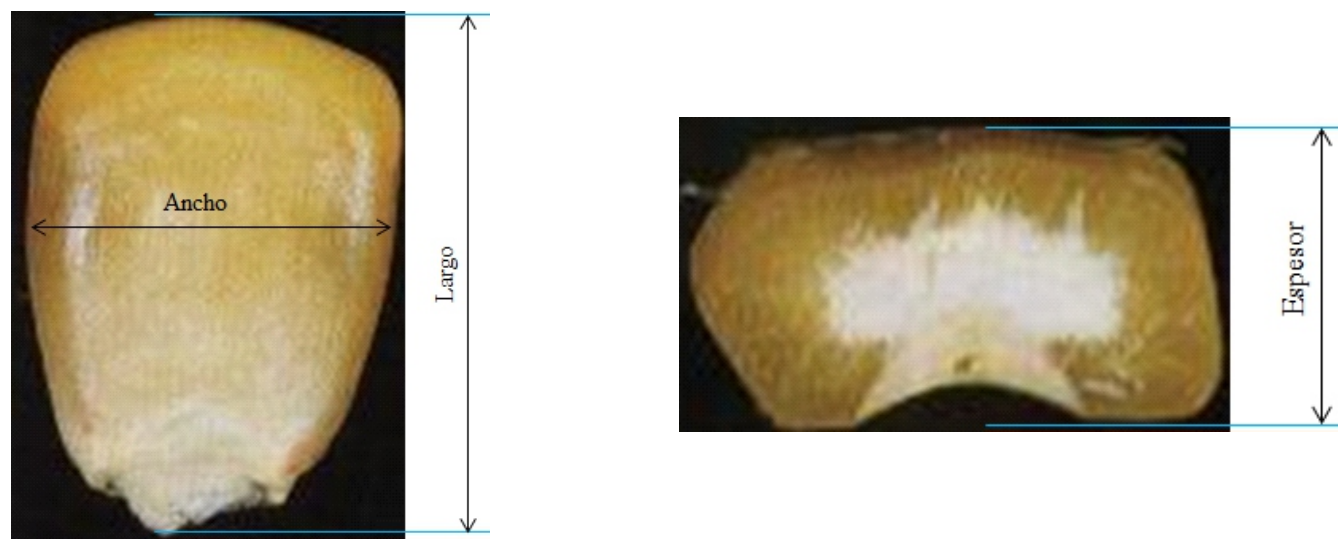

Figura 2. Dimensiones que caracterizan a la semilla de maíz.

aires a través de una malla metálica, logrando la separación vertical de las semillas, en función de su volumen, peso y textura, para posteriormente efectuar una separación horizontal de los estratos, de acuerdo a la inclinación transversal de la masa de semillas y a su movimiento transversal ascendente, que permite la separación de la masa de semillas en tres categorías, las muy densas, las de menor densidad y las intermedias (Oropeza et al., 2000).

\section{Tratamiento de semilla}

Por tratamiento de semilla se entiende la aplicación de alguna sustancia a la semilla, con el fin de protegerla del daño ocasionado por microorganismos e insectos. De acuerdo a Copeland (1976), el tratamiento químico ideal para la semilla debería ser altamente efectivo contra organismos patogénicos, relativamente no tóxico al cultivo, inofensivo a los humanos, animales domésticos, aún si es mal manipulado, estable por largos períodos de tiempo durante el almacenamiento de la semilla, fácil de usar y económicamente competitivo.

Desafortunadamente, ninguno de los productos químicos comúnmente usados en el tratamiento de semilla reúnen todos estos requisitos. En Latinoamérica existen algunos productos químicos que se deben utilizar justo antes de la siembra de la semilla, puesto que en cortos períodos de tiempo afectan negativamente el vigor y la capacidad de germinación de la misma.

Conforme a Copeland (1976), la normativa internacional exige que la semilla tratada sea identificada de dos maneras distintas: agregando un colorante al tratamiento, que contraste con el color de la semilla y colocando una etiqueta que indique el tipo de producto utilizado y el antídoto en caso de envenenamiento.

\section{Envasado y almacenamiento}

Normalmente, después que la semilla es cosechada y procesada debe ser almacenada y conservada durante un período de tiempo determinado, que puede ir desde algunos pocos meses, hasta uno o más años, tiempo en el cual es 
necesario su manutención dentro de condiciones ambientales tales, que no modifiquen sus características cualitativas y cuantitativas. Una vez fin a 1 i zado e 1 proceso de acondicionamiento de la semilla, está lista para ser envasada y almacenada.

Tanto el envasado, como el almacenamiento deben realizarse de manera apropiada para mantener las semillas en las mejores condiciones, con el fin de asegurar al máximo su poder germinativo y otros parámetros de calidad. Conforme a Oropeza et al. (2000), lo común es que el envasado se haga en sacos de papel multipliegos o de lámina de plásticos de espesor $2 \times 0,20$ $\mathrm{mm}$, de dimensiones aproximadas (llenos y horizontales) de $1,10 \times 0,30 \times 0,125 \mathrm{~m}$. La semilla generalmente se acumula en un silo-tolva, colocado sobre una balanza automática de la que cuelga un saco; una vez vaciada en el saco la cantidad correspondiente al peso elegido, el saco se suelta y cae en una cinta transportadora que lo conduce hasta la cosedora (Sandoval, 2009).

El arrumaje de los sacos debe realizarse sobre estivas, para evitar el contacto directo con el piso y separado de las paredes de los depósitos. Los lotes de cada ruma de sacos no deben sobrepasar el límite máximo permitido, de 25,000 kg y en la medida de lo posible, debe dejarse suficiente espacio entre las rumas, para ventilación, muestreo e inspección (Oropeza et al., 2000).

Oropeza et al. (2000), mencionan que en la condición de países tropicales, caracterizados por altas temperaturas y humedades ambientales variables, los depósitos deben reunir las siguientes condiciones:

a. Ser totalmente impermeables a líquidos y gases, para mantener el aire del depósito dentro de determinados límites.

b. Estar termoaislado y en lo posible, impermeabilizado, para evitar el ingreso del aire externo al depósito, para evitar la rehidratación de la semilla, que provocaría el rápido deterioro directo de ésta, al acelerar el proceso respiratorio e indirectamente, por el desarrollo de diferentes plagas asociadas a las semillas almacenadas.

c. Ser a prueba de insectos, roedores y de aves granívoras, que pueden ocasionar pérdidas significativas, tanto en la calidad, como en cantidad.

En la literatura existe suficiente información relacionada con los diseños de los depósitos, las plantas para procesamiento de semillas, así como también de los equipos requeridos para los diferentes procesos de acondicionamiento de las semillas de maíz (Copeland, 1976; Johnson, 1980; Oropeza et al., 2000).

Las etapas del procesamiento de las semillas antes descritas, incluyendo el desgrane, limpieza, clasificación por forma y tamaño, tratamiento con funguicida e insecticida, embolsado 
y almacenamiento están bien estandarizadas (Paliwal, 2001). En la figura 3, se presenta un flujograma que representa las diferentes operaciones en el proceso de acondicionamiento de la semilla.

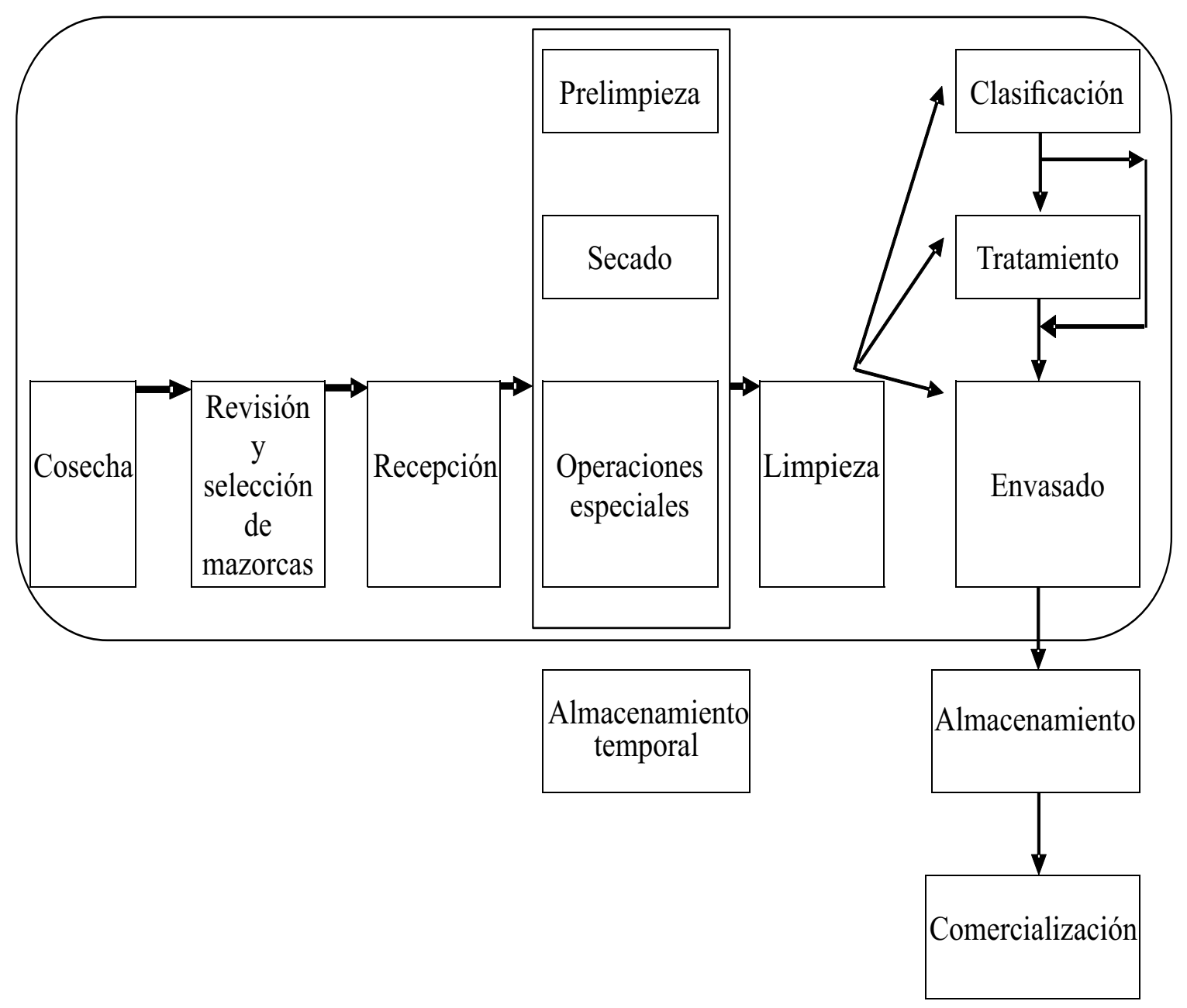

Figura 3. Flujograma de operaciones del acondicionamiento de semillas.

Adaptado de Copeland (1976). Diseño propio.

\section{CONCLUSIONES}

Una vez culminado el trabajo, se puede llegar a las siguientes conclusiones:

Reglamento de la Ley General de Semillas está orientado a tener un marco jurídico moderno e integrador, cuyo principal objetivo es fortalecer la actividad de la Autoridad en Semillas y otorgar transparencia y seguridad para los usuarios.

Las categorías de semillas se establecen con la finalidad de asegurar que en las distintas multiplicaciones se mantengan las características genéticas y fitosanitarias de los cultivares.

El sometimiento al proceso de certificación de semillas es un acto voluntario, para lo cual se requiere que los cultivares a ser multiplicados estén inscritos en el Registro de Cultivares 
Comerciales.

Por ser el maíz una planta alógama es indispensable tener un aislamiento adecuado, para evitar la contaminación entre los lotes de producción de semilla. La supervisión del proceso de producción de semilla es una actividad permanente, la cual es realizada tanto por personal técnico de la empresa o entidad productora, como por funcionarios oficiales de laAutoridad en Semillas.

La apariencia de la semilla no siempre representa un aspecto de calidad, pero afecta el mercadeo y la promoción, debido a que los productores no pueden apreciar en la semilla las bondades genéticas del cultivar que están comprando.

En el procesamiento de la semilla, posiblemente el secado sea una de las etapas más importante, debido a que a través de este proceso la semilla es llevada a un contenido de humedad óptimo para su conservación.

Las etapas del procesamiento de las semillas son actividades que están bien estandarizadas en el ámbito internacional.

\section{REFERENCIAS BIBLIOGRÁFICAS}

Agrawal, P. K.; B. D. Agarwal; P. Venkat Rao; J. Singh. (1998). Maize seed multiplication, conditioning, and storage, In: M.L. Morris, ed. Maize seed industries in developing countries. Boulder, CO, USA, Lynne Rienner Publishers.

Centro Internacional de Mejoramiento de Maíz y Trigo (CIMMYT). (1987).
1986 world maize facts and trends: the economics of commercial maize seed production in developing countries. Mexico, DF. Disponible en: http://repository.cimmyt.org/ xmlui/bitstream/handle/10883/699 /13573.pdf?sequence $=1$.

Centro Internacional de Mejoramiento de Maíz y Trigo (CIMMYT). 1994. 1993/94 world maize facts and trends: maize seed industries revisited: emerging role of the public and private sectors. Mexico, DF. Dis pon ib 1 e e n : http://repository.cimmyt.org/xmlui /bitstream/handle/10883/735/5345 9.pdf.

Cerda, E.; G. Aparicio; G. Fernández; S. González; A. Peña; R. Messina. (2000). Normas generales y específicas de certificación de semillas. Ministerio de Agricultura Servicio Agrícola y Ganadero. Departamento de Semillas. Santiago, Chile. Disponible en: http://www.sag.cl/sites/default/file s/NORMAS GENERALES SEM ILLAS.pdf.

Copeland, L. O. 1976. Principles of seed science and technology. Burgess Publishing Company. Mnneapolis, Minnesota. 369 p.

Dowswell, C. D.; R. L. Paliwal; R. P. Cantrell. 1996. Maize in the third world. Boulder, CO, USA, Westview Press.

El Peruano, 2008. Reglamento de la Ley General de Semillas. Decreto 
Supremo $\mathrm{N}^{\circ}$ 026-2008-AG. 9 p. D i s p o i b l e e n : https://www.serfor.gob.pe/pdf/normativi $\mathrm{dad} / 2008 /$ decresup/DS $\% 20 \mathrm{~N} \% \mathrm{C} 3 \% 82 \%$ C $2 \%$ B $0 \% \begin{array}{llllllllll} & 0 & 0 & 0 & 2 & 6 & -2 & 0 & 0 & 8\end{array}-$ AG(Ley\%20General\%20de\%20Semillas ).pdf.

Food and Agriculture Organization of the United Nations (FAO). (2005). Producción artisanal de semilla de maíz para el pequeño productor. Ficha Técnica. Technologies and practices for small agricultural producers (TECA). 4 p. Disponible en: ht tp://www.fao.org/ 3/CA3410ES/ca3410es.pdf

Granados, G. 2001. Manejo postcosecha. En: El maíz en los trópicos: mejoramiento y producción. Paliwal, R. L.; G. Granados; H. R. Laffite; A.D. Violic (Edes.). FAO, CIMMYT. Roma, 2001. Disponible en: https://curlacavunah. files.wordpress.com/2010/04/elmaiz-en-los-tropicos.pdf.

Hallauer, A. R.; M. J. Carena; J. B. Miranda Filho. 2010. Quantitative genetics in maize breeding. 3rd ed. London: Springer. 663 p.

Instituto Nacional de Innovación Agraria (INIA). 2009. Resolución Jefatural $\mathrm{N}^{\circ}$ 00166-2009 - INIA. Normas Producción de Semilla. Disponible en: http://www.inia.gob.pe/wpcontent/uploads/LegislacionSemill as/RJ-166-2009.pdf

Instituto Nacional de Innovación Agraria (INIA). 2013. Resolución Jefatural
$\mathrm{N}^{\circ}$ 00057-2013 - INIA. Normas para la Producción de Semilla de Maíz Amiláceo. Disponible en: http://www.inia.gob.pe/wpcontent/uploads/LegislacionSemill a s / R J - $00057-2013$ INIA_NormaMaizAmilaceo.pdf.

Johnson, D. (1980). Succesful seed programs; A planing and management guide. Westview Press /Boulder, Colorado. 302 p.

López-Pereira, M. A.; M. P. Filippello. (1995). Emerging roles of the public and private sectors of maize seed industries in the developing world. CIMMYT Economics Working Paper 95/01, Mexico, DF.

Martínez, H. 1982. Fragmento Histórico de la Producción de Semillas. FONAIAP Divulga No. 4. MayoJunio 1982. Disponible en: http://sian.inia.gob.ve/repositorio/r evistas_tec/FonaiapDivulga/fd04/t exto/historiadesemilla.htm.

Ministerio de Agricultura y Cría (MAC). 1986. Normas Generales sobre Semillas. En: Gaceta Oficial $\mathrm{N}^{\circ}$ 33.456 del 24 de abril de 1986. Caracas, Venezuela. Disponible en: https://research.cip.cgiar.org/confl uence/download/attachments/1792 4178/Reglementacion+Venezuela. pdf.

Ministerio de Agricultura y Riego ( $\left.\begin{array}{lllllll}\mathrm{M} & \mathrm{I} & \mathrm{N} & \mathrm{A} & \mathrm{G} & \mathrm{R} & \mathrm{I}\end{array}\right)$. 2012.COMUNICADO: Se aprobó el Reglamento de la Ley General de Semillas Decreto Supremo No $006-$ 
2012-AG. 38 p. Disponible en:

http://extwprlegs1.fao.org/docs/pdf/per1

13605txt.pdf.

Oropeza, H.; B. Mazzani. (1997).

Evolución tecnológica del maíz en

Venezuela: Selección de cultivares

y producción de semilla. Fundación

Polar; Fondo Nacional de

Investigaciones Agropecuarias

(FONAIAP), Caracas, Venezuela.

$50 \mathrm{p}$.

Oropeza, H.; C. Márquez; D. Nuñez.

(2000). Tecnología de la producción de semillas. En: El Maíz en Venezuela. Fontana, H. y C. González (Edes.). Fundación Polar. p. 407-437.

Paliwal, R. L. (2001). Producción de semilla. En: El maíz en los trópicos: mejoramiento y producción. Paliwal, R. L.; G. Granados; H. R. Laffite; A. D. Violic (Edes.). FAO, CIMMYT. Roma, 2001. Disponible e $\mathrm{n}$

https://curlacavunah.files.wordpres s.com/2010/04/el-maiz-en-lostropicos.pdf.

Programa Nacional de Semillas (PNS). (2000). Norma general sobre semilla de especies agrícolas $-\mathrm{R}$. M. 121 del $19 / 12 / 00$. Bolivia. D i s p o n ible e n : http://www.senapi.gob.bo/snpDow nload/MarcoLegalVigente/RS_1.p df.

Sandoval, A. (2009). Almacenamiento de semillas. Centro de semillas y árboles forestales. Facultad de
Ciencias Forestales. Chile. D i s p o n ible e n : http://www.cesaf.uchile.cl/cesaf/n1 4/1.html>.

The maize Program. 1999. Development, maintenance, and seed multiplication of open-pollinated maize varieties. $2^{\text {nd }}$.Edition. México, D. F. CIMMYT. Dis ponible e n : https://repository.cimmyt.org/bitstr eam/handle/10883/758/67323.pdf? sequence $=4 \&$ is Allowed $=\mathrm{y}$

United States Department of Agriculture (USDA). (2009). Understanding seed certification and seed labels. Technical notes. Natural Resources Conservation Service. Alexandria, Louisiana, USA. Disponible en: http://www.nrcs.usda.gov/Internet/ FSE_PLANTMATERIALS/public ations/lapmctn9030.pdf.

Wych, R. D. (1988). Production of hybrid seed corn. En: Sprague, G, F. y J. W. Dudley. (Edes.). Corn and corn Improvement. Third Edition, Madison, WI. p. 565-607.

\section{CORRESPONDENCIA:}

Dr. Pedro Garcia Mendoza pejogam@gmail.com 